\title{
Research article \\ Effect of L-tryptophan on intestinal glucagon like peptide-1(GLP-1) in streptozotocin (stz) induced diabetic rat model
}

\author{
Vineetha K. K. ${ }^{1}$, Gayathri M. Rao ${ }^{1}$, K. Ashok Prabhu ${ }^{1}$, Vinodchandran ${ }^{1}$, Kavyashree ${ }^{1}$, Aradhana Marathe ${ }^{1}$, Vandana \\ Blossom $^{2}$ \\ ${ }^{1}$ Department of Biochemistry, ${ }^{2}$ Department of Anatomy, Center for Basic Sciences, Kasturba Medical College, Mangalore, \\ Manipal Academy of Higher Education, Manipal, Karnataka, India
}

(Received: April $2021 \quad$ Revised: May $2021 \quad$ Accepted: June 2021)

Corresponding author: Gayathri M. Rao. Email: gayathri.rao@manipal.edu

\begin{abstract}
Introduction and Aim: The management of diabetes is a great challenge in this era due to several factors. The financial burden of the medications and the complications of diabetes create management demanding especially in the Indian population. A high protein-rich diet is beneficial in controlling hyperglycemia in diabetic patients. Different amino acids play roles in reducing blood glucose levels in diabetes. Certain amino acids trigger hypoglycemia in diabetes by inducing the gut hormone, Glucagon-like peptide -1(GLP-1) secretion. L-tryptophan is one among them and is present in many of the food items like poultry, beans, seeds, nuts, etc., This study using albino rats focuses on the effect of L-tryptophan on intestinal GLP-1 secretion in diabetic rats by evaluating the blood glucose level, intestinal GLP-1, and intestinal histology of diabetic rats after tryptophan administration.
\end{abstract}

Materials and Methods: Single dose of intraperitoneal injection of STZ $(50 \mathrm{mg} / \mathrm{kg})$ used to develop diabetic model and orogastric gavage of tryptophan $(50 \mathrm{mg} / \mathrm{kg}$ ) was done. Intestinal GLP-1 and blood glucose assay and intestinal histology were the parameters studied.

Results: The results showed a significant reduction in blood glucose level and an increased secretion of intestinal GLP-1 $(\mathrm{p}=0.001)$ in diabetic rats by tryptophan administration and recovery was seen in intestinal histology. In conclusion, in our study, the administration of L- tryptophan in diabetic rats induced a hypoglycemic effect by GLP-1 secretion and restored normal histology.

Conclusion: Tryptophan administration showed hypoglycemic effect on diabetic rats as the blood glucose level was reverted to normal and the hypoglycemic effect of L-tryptophan in diabetic rats could be due to increased GLP-1 secretion.

Keywords: Blood glucose; L-tryptophan; hypoglycemia; glucagon-like peptide-1(GLP-1).

\section{INTRODUCTION}

$\mathrm{D}$ iabetes mellitus (DM), a chronic metabolic disease characterized by high blood glucose level, classified as Type 1 and Type 2, either due to insulin deficiency or insulin resistance, respectively. According to the latest data from $\mathrm{WHO}$, globally 422 million adults are living with diabetes $(1,2)$. Diet planning appears to play a beneficial role in the management of DM. A high protein and low carbohydrate diet together induce a favorable effect in diabetic patients since it increases the glucose energy expenditure and reduces the burden on pancreatic $\beta$-cells (3).

Different constituent amino acids in a protein-rich diet exert varied effects on the blood glucose level. Tryptophan, an essential aromatic amino acid acts as a building block for many essential biomolecules, including enzymes, structural proteins, neurotransmitters like serotonin and melatonin (4-7). Tryptophan potentially stimulates gastrointestinal functions. The neurotransmitter serotonin synthesized from tryptophan plays an important role in the regulation of GI motility and appetite control in coordination with the hypothalamus (8).

Since insulin and incretin hormones shows a significant role in post-prandial glycemic regulation. Dietary polypeptides and amino acids are shown to act as insulin and incretin hormone secretagogues and thereby regulate the postprandial glycemia in animals and humans $(9,10)$. L-tryptophan plays an important role in diabetes management by inhibiting intestinal absorption of glucose and stimulating its cellular uptake $(11,12)$. Study report showed that tryptophan metabolites could inhibit hepatic gluconeogenesis from lactate (13). Hormones released from both islets of the pancreas and gastrointestinal glands have been shown to take part in the regulation of glucose metabolism and thereby in diabetes pathogenesis. Glucagon-like peptide 1(GLP-1) is a gut hormone and an incretin family member, is synthesized and secreted by intestinal enteroendocrine L-cells, upon food ingestion as well as by certain neurons in the nucleus of the solitary tract of the brainstem.GLP-1 is a transcription product of the proglucagon gene (14-16). It has been shown that GLP-1 stimulates glucose-dependent insulin 
secretion, inhibits glucagon, reduces appetite, and delays gastric emptying. GLP-1 based treatment in diabetes has shown some beneficial effects by stimulating $\beta$-cell proliferation and decreasing the rate of $\beta$-cell apoptosis (17).

The present study undertaken to investigate the effect of L-tryptophan on blood glucose and GLP-1 levels in streptozotocin (STZ) -induced diabetic rat model.

\section{MATERIALS AND METHODS}

\section{Subjects}

Adult albino rats of Wistar strain $(n=24)$ were used for the study. Animals weighing about 200-250 g were obtained from Central Animal House, Kasturba Medical College, Mangalore, India. The study protocols were approved by the Institutional Animal Ethics Committee, (KMC/MNG/IAEC/03-2019), Kasturba Medical College, Mangalore.

\section{Study design and protocol}

\section{Diabetic Model}

By single dose of Streptozotocin -i.p. $50 \mathrm{mg} / \mathrm{kg}$ body weight.

\section{Tryptophan treatment}

$50 \mathrm{mg} / \mathrm{kg}$ body weight of L- tryptophan by orogastric gavage, once in a day for 10 days.

Animals $(n=24)$ were acclimatized for a week under standard conditions - standard pellet and water ad libitum. They were divided into 4 groups ( $n=6 /$ group).

\section{Group 1: Normal control (NC)}

Group 2: Diabetic control - Treated with single dose of streptozotocin (i.p $50 \mathrm{mg} / \mathrm{kg}$ body weight)

Group 3: Tryptophan control (TC) rats treated with $50 \mathrm{mg} / \mathrm{kg}$ body weight of L- tryptophan by orogastric gavage, once in a day for 10 days

Group 4: Tryptophan administered diabetic rats (D+T): Tryptophan $(50 \mathrm{mg} / \mathrm{kg}$ body weight) were administered to the diabetic rats orally once in a day for next 10 days.

Estimation of blood glucose was done by collecting the blood sample from the tail vein between 48-72 hours of STZ administration. The animals with blood glucose levels above $250 \mathrm{mg} / \mathrm{dl}$ were considered diabetic. Thereafter, blood glucose level was estimated by tail prick, once in 4 days using a glucometer. The animals were sacrificed by ketamine overdose after 10 days. The collected small intestine tissue was analyzed for GLP-1 analysis and histological studies. A $10 \%$ homogenate was prepared in saline phosphate buffer, centrifuged and the supernatant was stored at $-20^{\circ} \mathrm{C}$ for estimation of intestinal GLP-1.

\section{Measurements}

Blood glucose estimation using glucometer by collecting blood from the tail vein (18) during the study.10\% Intestinal homogenate GLP-1 was assayed from the by Enzyme-linked immunosorbent assay (ELISA) (Weldon Biotech India Pvt. Ltd).

Histological study: Tissues fixed in $10 \%$ neutral buffered formalin solution, paraffin sections of $5 \mu \mathrm{m}$ thickness stained with hematoxylin-eosin (H-E) and examined on a light microscope.

\section{Statistical analysis}

The analysis was done using the EZR statistical package. The paired sample t-test to compare the dependent groups, independent-sample t-test between independent groups. ANOVA was done to compare means between independent groups, $\mathrm{p}$-value of $<0.05$ was considered as significant.

\section{RESULTS}

Group 4 - Tryptophan treated diabetic rats, showed a marked decrease in blood glucose, as highly significant $(\mathrm{p}=0.001)$ change when compared to group 2 (369 \pm 83.4$)$ (fig.1). Intestinal GLP-1 secretion significantly increased $(\mathrm{p}<0.01)$, among group 4 when compared with other groups. However, the increase mild increase in GLP-1 secretion was seen when compared between normal control and diabetic control $(84.3 \pm 11.6$ and $87.4 \pm 4.6$ respectively) and significant increase in GLP-1 secretion between normal control and tryptophan control (84.3 \pm 11.6 and 98.5 \pm 7.03 , $\mathrm{p}<0.05$, fig. 2$)$. Intestinal histology showed slight mucosal destruction, which was evident from the thinning of the submucosal layer and slightly distorted villi. Recovery has been observed in diabetic group treated with tryptophan, which was evident from the crowding of the cells, indicated regeneration (fig.3).

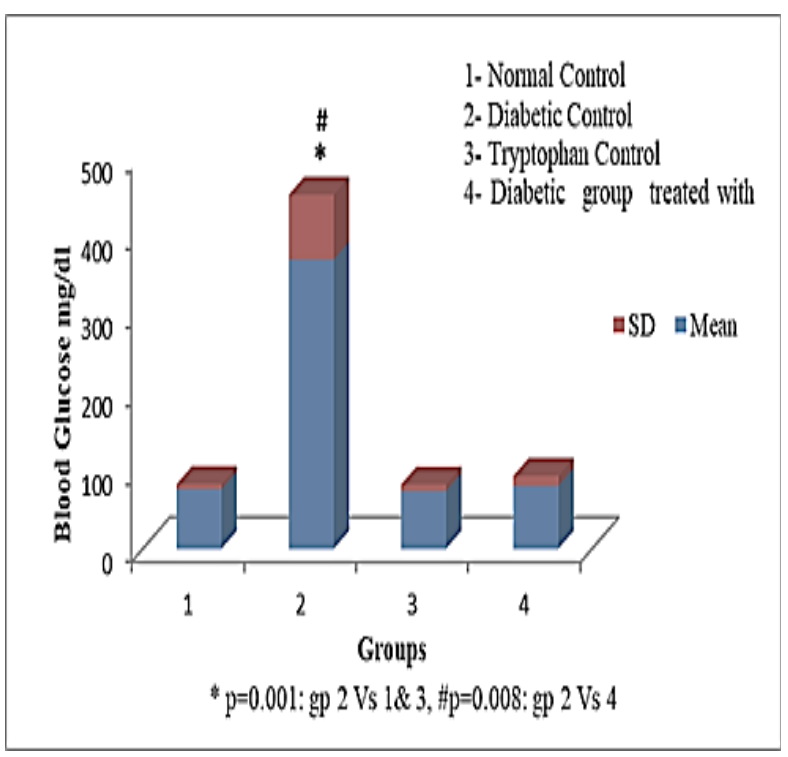

Fig. 1: Blood glucose level (mg/dl) at the time of dissection 


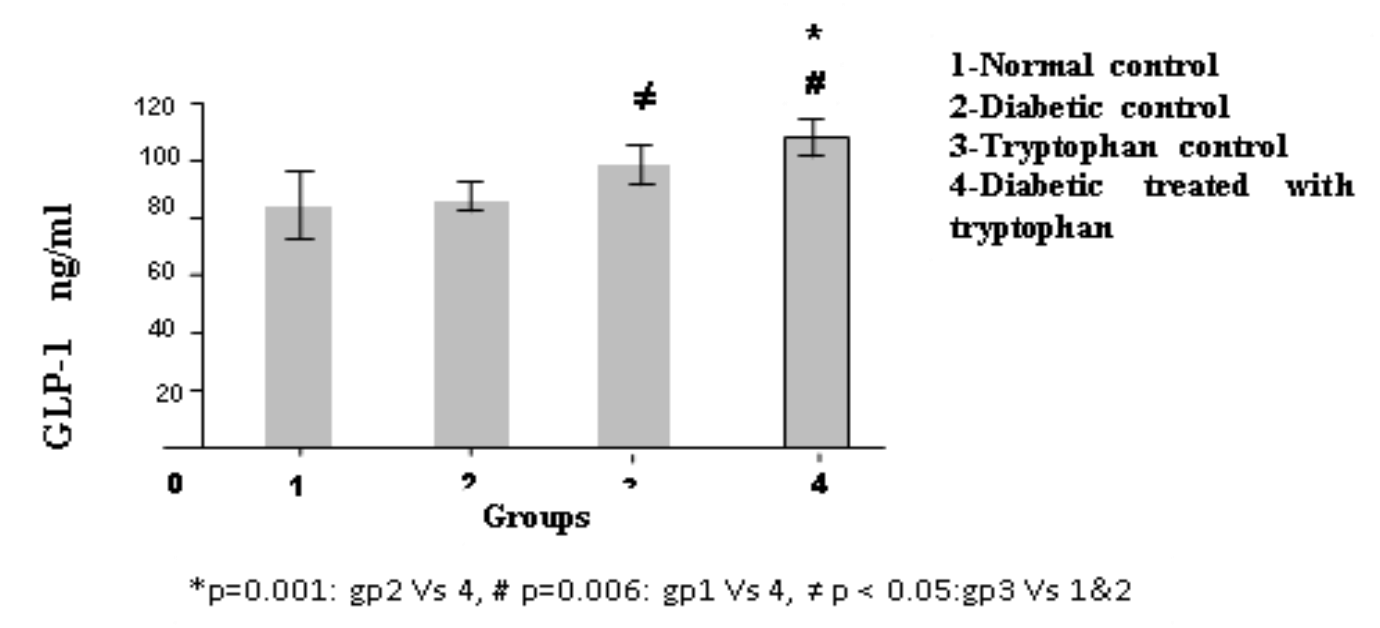

Fig. 2: Intestinal GLP-1 (ng/ml)
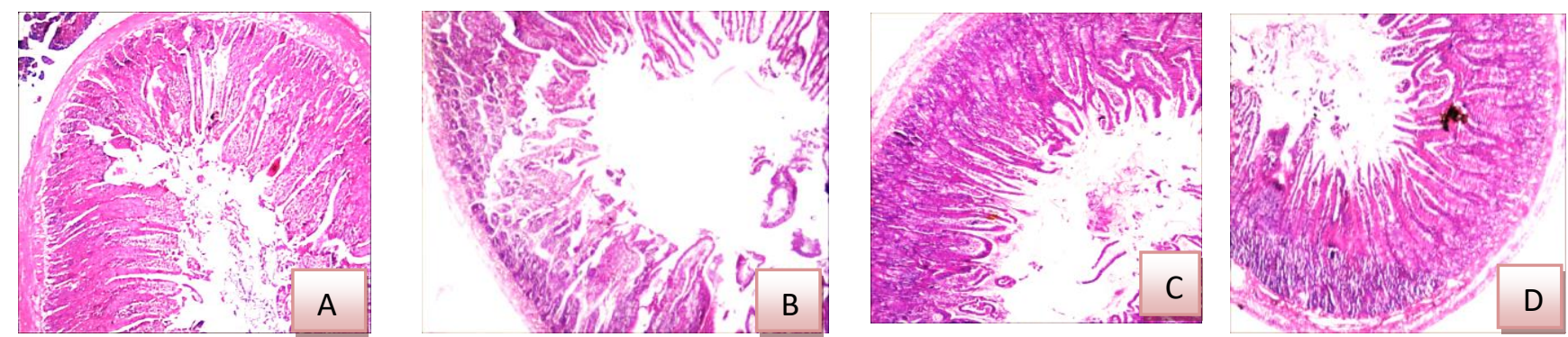

Fig. 3: Intestinal histology A) Normal control B) Diabetic control: slightly destructed villi and submucosal layer thinning C) Tryptophan control: showed a normal histology D) Diabetic treated with tryptophan: crowding of cells and cellular regeneration.

\section{DISCUSSION}

Diabetes results in altered metabolic processes. Diet management has an important role in diabetes control. A high protein-containing diet has some effects on glycemic control especially in diabetic patients (18). Study reports shows that high protein diet helps to reduce total energy intake and body weight. This was described using various mechanisms including the stimulation of insulin release, postprandial thermogenesis; intestinal gluconeogenesis, etc., $(19,20)$. Several amino acids shown to have a stimulatory effect on insulin release. It has been reported that tryptamine formed from tryptophan inhibited intestinal absorption of glucose and increased the glucose associated energy expenditure in diabetic rats after oral glucose administration (12).

In healthy as well as in diabetic patients, the regulation of food intake and postprandial blood glucose maintenance is by delaying the process of gastric emptying and gut hormone release after the ingestion of nutrients. Ullrich et al., (7) studied the effect of tryptophan on blood glucose after administering nutrient drink and reported an attenuation of the postprandial rise in blood glucose. However intra-gastric or oral administration of tryptophan has been reported to have a hypoglycemic effect with increased plasma tryptophan level $(7,21)$. In this study, we observed an improvement in glycemic control after tryptophan administration. Our results agree with previous similar studies. Syah et al., (22) have reported an improvement in blood pressure, blood glucose, and insulin after oral tryptophan administration in stroke-prone hypertensive rats. Their study demonstrated a hypoglycemic effect both after a single dose as well as after continuous administration of tryptophan. The formation of serotonin from tryptophan may explain the mechanism behind the reduction in blood glucose levels (22). It was reported that hepatic gluconeogenesis was inhibited by tryptophan at the level of phosphoenolpyruvate formation (13). This can result in setting control over fasting blood glucose levels. Intra-duodenal or intravenous infusion of tryptophan is also shown to have stimulatory action on insulin release $(21,23)$.

A study by Acar et al., reported an increase in GLP-1 secretion after intra-duodenal tryptophan infusion (24). The stimulatory effect of tryptophan on GLP-1 secretion could be due to various mechanisms. The calcium-sensing receptor (CaSR) of the enteroendocrine cells is a major extracellular calcium sensor. It is considered as a physiological sensor of amino acid for the gut hormone release. Aromatic Lamino acids were found to regulate calcium, an essential ligand for CaSR (24-26). GPR142, a specific G-protein coupled receptor, expressed in pancreatic $\beta$ cells and gastrointestinal tract, accepted as an amino acid receptor (9).

The observed hypoglycemic effect of the tryptophan in diabetic rats could be due to the agonistic action of tryptophan on GPR142. A deficient GLP-1 secretion 
was reported among patients with long-standing DM and poor glycemic control $\left(\mathrm{HbA}_{1} \mathrm{c} \sim 9.2 \%\right)$ in earlier studies $(27,28)$. Recent studies reported that there was not much difference in GLP-1 level among patients with mild $\mathrm{DM}\left(\mathrm{HbA}_{1} \mathrm{c} \sim 6.8 \%\right)$, impaired glucose tolerance, and normal glucose tolerance. These reports confirm that GLP-1 secretion in diabetic patients may depend on their glycemic status, intake of medications, other metabolic and hormonal abnormalities, which will worsen their glycemic control (17).

These findings may explain the hypoglycemic effect of L- tryptophan that was observed in the present study. The Intestinal histology study also supports the recovery after tryptophan administration. Mild to moderate level of villi destruction and thinning of the submucosal layer has been observed in diabetic rats. Nagilla and Reddy (29) have reported a moderate level of alterations in intestinal histology with excess proliferation and edema of villi in diabetic rats. An alteration in the histology of small intestine is also one more observation during the study. However, a marked recovery is observed in the histology after tryptophan administration.

\section{CONCLUSION}

Tryptophan administration showed hypoglycemic effect on diabetic rats and blood glucose level was reverted to normal. The present study proposes that the hypoglycemic effect of L-tryptophan in diabetic rats could be due to increased GLP-1 secretion. Histological study of intestine revealed a recovery after tryptophan administration in diabetic rats. This study put forward a possible role for tryptophan as a dietary therapeutic intervention to treat DM. However, an extensive study on diabetic rats for a longer time is required to substantiate the observation.

\section{CONFLICT OF INTEREST}

Authors declare no conflict of interest.

\section{REFERENCES}

1. World Health Organization. http:/www.who.int/ mediacenter/factsheet/diabete $\mathrm{s} / 2018$.

2. Shrivastava, S. R., Shrivastava, P. S., Ramasamy, J. Role of self-care in management of diabetes mellitus. J Diabetes Metab Disord. 2013; 5: 12(1): 14. doi: 10.1186/2251-658112-14. PMID: 23497559; PMCID: PMC3599009.

3. Farnsworth, E., Luscombe, N.D., Noakes, M., Wittert, G., Argyiou, E., Clifton. P. M. Effect of a high-protein, energyrestricted diet on body composition, glycemic control, and lipid concentrations in overweight and obese hyperinsulinemic men and women. The American Journal of Clinical Nutrition. 2003; 78(1): 31-39.

4. Wada, K., Yata, S., Akimitsu, O., Krejci, M., Noji, T., Nakade, M, et al., A tryptophan-rich breakfast and exposure to light with low color temperature at night improve sleep and salivary melatonin level in Japanese students. J Circadian Rhythms. 2013; 25(1): 4. doi: 10.1186/17403391-11-4. eCollection

5. Kałużna-Czaplińska, J., Gątarek, P., Chirumbolo, S., Chartrand, M. S., Bjørklund, G. How important is tryptophan in human health? Critical Reviews in Food Science and Nutrition. 2017; 59(1): 72-88.

6. Friedman, M. Analysis, Nutrition, and Health Benefits of Tryptophan. International Journal of Tryptophan Research. 2018; 11: 11. 7864691880228.

7. Ullrich, S. S., Fitzgerald, P. C. E., Giesbertz, P., Steinert, R. E., Horowitz, M., Feinle-Bisset, C. Effects of Intragastric Administration of Tryptophan on the Blood Glucose Response to a Nutrient Drink and Energy Intake, in Lean and Obese Men. Nutrients. 2018; 10(4): 463. Published 2018 Apr 8. doi:10.3390/nu10040463

8. Palego, L., Betti, L., Rossi, A., Giannaccini, G. Tryptophan Biochemistry: Structural, Nutritional, Metabolic, and Medical Aspects in Humans. Journal of Amino Acids. 2016: 2016: 1-13.

9. Lin, H. V., Efanov, A. M., Fang, X., Beavers, L. S., Wang, X., Wang, J., et al., GPR142 Controls Tryptophan-Induced Insulin and Incretin Hormone Secretion to Improve Glucose Metabolism. PLoS One. 2016 Jun 20; 11(6): e0157298. doi: 10.1371/journal.pone.0157298. PMID: 27322810; PMCID: PMC4920590.

10. Gribble, F. M., Meek, C. L., Reimann, F. Targeted intestinal delivery of incretin secretagogues-towards new diabetes and obesity therapies. Peptides. 2018: 100: 68-74.

11. Steinert, R. E., Ullrich, S.S., Geary, N., Asarian, L., Bueter, M., Horowitz, M., et al., Comparative effects of intraduodenal amino acid infusions on food intake and gut hormone release in healthy males. Physiol Rep. 2017; 5(21): e13492. doi:10.14814/phy2.13492

12. Inubushi, T., Kamemura, N., Oda, M., Sakurai, J., Nakaya, Y., Harada, N., et al., L-Tryptophan suppresses rise in blood glucose and preserves insulin secretion in type-2 diabetes mellitus rats. Journal of Nutritional Science and Vitaminology. 2012; 58(6): 415-422.

13. Katsunori, N., Takao, K., Mari, M. A tryptophan hydroxylase inhibitor increases hepatic FGF21 production and decreases hepatic gluconeogenesis independently of insulin in $\mathrm{db} / \mathrm{db}$ mice neuropsychiatry (London) 2018: 8(4): 1476-1481.

14. Drucker, D. J. The biology of incretin hormones. Cell Metabolism. 2006; 3(3): 153-165.

15. Boer, G. A., Holst, J. J. Incretin hormones and type 2 diabetes-mechanistic insights and therapeutic approaches. Biology. 2020; 9:473..https://doi.org/10.3390/bi ology9120473

16. Holst, J. J. The physiology of glucagon-like peptide 1 . Physiological Reviews. 2007; 87(4): 1409-1439.

17. Sodai, K., Yanyan, L., Katsumi, I., Hitoshi, K., Yutaka, S., Daisuke, Y. A Review of Recent Findings on Meal Sequence: An Attractive Dietary Approach to Prevention and Management of Type 2 Diabetes. Nutrients. 2020; 12: 2502; doi:10.3390/nu12092502

18. Jiao, L., Xiujuan, S., Juan, W., Song, J., Lei, X., Guotong, X., Lixia, L. Comprehensive experiment-clinical biochemistry: Determination of blood glucose and triglycerides in normal and diabetic rats. Biochemistry and Molecular Biology Education. 2014Dec17; 43(1): 47-51.

19. Gannon, M. C., Nuttall, F. Q. Effect of a high-protein, lowcarbohydrate diet on blood glucose control in people with type 2 diabetes. Diabetes. 2004; 53(9): 2375-2382.

20. Larsen, R. N., Mann, N. J, Maclean, E., Shaw, J. E. The effect of high-protein, low-carbohydrate diets in the treatment of type 2 diabetes: a 12-month randomised controlled trial. Diabetologia. 2011; 54(4): 731-740.

21. Steinert, E., Luscombe-Marsh, N. D., Little, T. J., Standfield, S., Otto, B., Horowitz, M. Effects of intraduodenal infusion of l-tryptophan on ad libitum eating, antropyloroduodenal motility, glycemia, insulinemia, and gut peptide secretion in healthy men. The Journal of Clinical Endocrinology \& Metabolism. 2014: 99(9): 3275-3284.

22. Ardiansyah, S., Shirakawa, H., Inagawa, Y., Koseki, T., Komai, M. Regulation of blood pressure and glucose metabolism induced by L-tryptophan in stroke-prone 
spontaneously hypertensive rats. Nutrition \& metabolism. 2011 Dec; 8(1): 1-7.

23. Tingting, Z., Changhong, L. Mecahnisms of amino acidstimulated insulin secretion in congenital hyperinsulinism. Acta Biochmimica et Biophysica Sinica. 2013; 45(1): 36-43.

24. Acar, I., Cetinkaya, A., Lay, I., Ileri-Gurel, E. The role of calcium sensing receptors in GLP-1 and PYY secretion after acute intraduodenal administration of L-Tryptophan in rats. Nutritional Neuroscience. 2018; 1-9.

25. Wang, J., Carrillo, J.J., Lin, H.V. GPR142 Agonists Stimulate Glucose-Dependent Insulin Secretion via GqDependent Signaling. PLoS ONE. 2016: 11(4): e0154452. https://doi.org/10.1371/journal.pone.0154452

26. Ueda, Y., Iwakura, H., Bando, M., Doi, A., Ariyasu, H., Inaba, H., et al., Differential role of GPR142 in tryptophanmediated enhancement of insulin secretion in obese and lean mice. PloS one. 2018; 13(6): e0198762. https://doi.org/10.1371/journal.pone.0198762

27. Nauck, M. A., Glucagon-like Peptide 1 (GLP-1) in the treatment of diabetes. Hormone and Metabolic Research. 2004; 36(11/12): 852-858.

28. Toft-Nielsen, M. B., Damholt, M. B., Madsbad, S., Hilsted, L. M., Hughes, T. E., Michelsen, B. K., et al., Determinants of the impaired secretion of glucagon-like peptide- 1 in type 2 diabetic patients. The Journal of Clinical Endocrinology \& Metabolism. 2001; 86(8): 3717-3723.

29. Nagilla, B., Reddy, K. P. Effect of methanolic leaf extract of Coccinia grandis on histopathology of Liver, Kidney and Intestine of STZ-induced diabetic Wistar rats. Research Journal of Pharmaceutical, Biological and Chemical Sciences. 2017: 727-737. 